\title{
Behavior estimation for a complete framework for human motion prediction in crowded environments
}

\author{
Gonzalo Ferrer and Alberto Sanfeliu \\ Institut de Robòtica i Informàtica Industrial (CSIC-UPC), Llorens Artigas 4-6, 08028 Barcelona, Spain. \\ \{gferrer, sanfeliu\}@iri.upc.edu
}

\begin{abstract}
In the present work, we propose and validate a complete probabilistic framework for human motion prediction in urban or social environments. Additionally, we formulate a powerful and useful tool: the human motion behavior estimator. Three different basic behaviors have been detected: Aware, Balanced and Unaware. Our approach is based on the Social Force Model (SFM) and the intentionality prediction BHMIP. The main contribution of the present work is to make use of the behavior estimator for formulating a reliable prediction framework of human trajectories under the influence of dynamic crowds, robots, and in general any moving obstacle. Accordingly, we have demonstrated the great performance of our long-term prediction algorithm, in real scenarios, comparing to other prediction methods.
\end{abstract}

\section{INTRODUCTION}

A great interest in human motion prediction has rapidly grown among the robotic community. In this paper, we present a complete probabilistic framework for human motion prediction. The aforementioned framework consists of a prediction algorithm, the intentionality predictor, and the behavior estimator. We analyze human trajectories under the influence of dynamic crowded and heterogeneous environments, where there are moving people, moving objects, robots and also static obstacles.

The Social Force Model (SFM) [1] presents accuracy limitations when performing micro-prediction of human motion. The works such as [2] have proposed a learning-based motion prediction approach which outperforms the SFM. Nonetheless, the present work expects to push the human motion prediction paradigm a little further with the help of an improved SFM. We suggest to estimate the interaction parameters described in the SFM for every target to improve greatly the prediction performance. Every person is different and behaves differently in equal situations, especially when robots appear. This serves as a motivation to obtain the estimator of human behavior for enhancing the human motion prediction.

The prediction of human behavior is not an easy endeavor. There exist a wide variety of approaches such as the Social Force Model (SFM), heading heuristics [3], and learningbased approaches [2], [4]. It is still unclear which approach works better since the calibration of models may be tricky and leads to contradictory conclusions.

Work supported by the Spanish Ministry of Science and Innovation under project RobTaskCoop (DPI2010-17112).

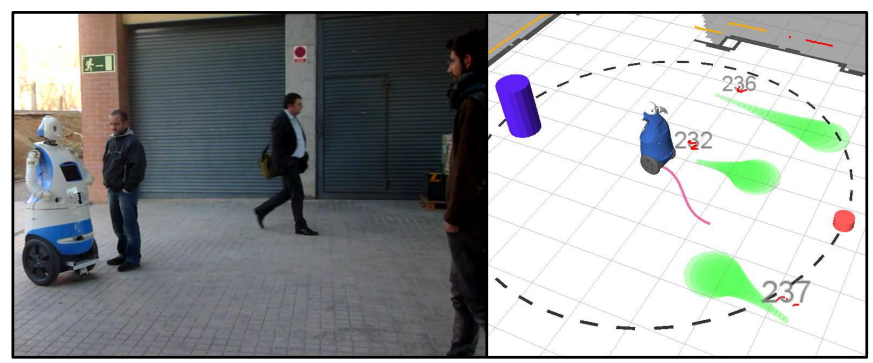

Fig. 1. Urban environment corresponding to the BRL, where part of the experiments were carried out. Prediction position covariances are drawn as green ellipses projected and superposed in the plane.

The work of [5] has treated the problem by considering a collision time which determines the magnitude of the interacting force. Nevertheless, this model is not able to explain why different people behave differently, under the same configuration of the scene.

In [6], the human motion prediction is obtained using control criteria and the human trajectories are generated in controlled environments.

In [7], the authors have proposed a place-dependent prediction method in which they analyze a collection of people's trajectories in indoor environments. They cluster those motion trajectories, by using the Expectation-Maximization algorithm, and once they learn these primitive trajectories, they classify human motion trajectories.

Using heuristics and geometric criteria [8] has proposed a geometric-based method for human motion prediction that uses human motion intentionality in terms of goals.

A work by [9] uses both place dependences and geometric criteria. The authors have proposed to use a reward function to generate the optimal paths towards a destination. This method can also be used as for modeling of route preferences as for inferring destinations.

In the remainder of the paper, we discuss the human prediction problem in Section II and we present the basic formulation. In section III, we describe the behavior estimation algorithm, one of the main contributions of the present paper, and we discuss the methods to train the estimator. In Section IV we propose a probabilistic human trajectory predictor, by making use of intentionality and human-behavior information. The experimental results and conclusions are presented in sections V and VI, respectively. 


\section{PROBlem OVERVIEW}

In this section, we will present the requirements to formulate a successful prediction framework. We will also present the tools that permit us to enhance the prediction performance, such as the Bayesian Human Motion Intentionality Predictor (BHMIP) [10] or the Social-Force Model (SFM). As we will demonstrate later, additional estimations of human parameters, like intention or behavior, clearly enhances the accuracy of the prediction.

In brief, we present the basic formulation necessary to analyze real trajectories. Let

$$
\boldsymbol{X}_{n}(t)=\left\{\boldsymbol{x}_{n}\left(t_{0}\right), \boldsymbol{x}_{n}\left(t_{0}+1\right), \ldots, \boldsymbol{x}_{n}(t)\right\}
$$

be a set of positions (people detections) where each point $\boldsymbol{x}_{n}(t)=[x(t), y(t)]_{n}$ is the position at time $t$ of the $n$th trajectory with respect to the world reference frame. Each point has its corresponding covariance $\Sigma_{n}(t)$. The joint set of trajectories is defined as

$$
\boldsymbol{X}(t)=\left\{\boldsymbol{X}_{1}(t), \boldsymbol{X}_{2}(t), \ldots, \boldsymbol{X}_{N}(t)\right\}
$$

Moreover, we define a set of destination positions $\boldsymbol{D}=$ $\left\{D_{1}, D_{2}, \ldots, D_{M}\right\}$, that indicates interesting positions in a scene that people might go to.

We infer the probability of the destination $P\left(\mathcal{D}_{n}(t)=\right.$ $\left.d_{m} \mid \boldsymbol{X}_{n}(t)\right)$, where $\mathcal{D}_{n}(t)$ is the inner intention for the $n$th person to reach the destination $D_{m}$, and can be any of the destinations $\boldsymbol{D}$ in the scene. For further details, see [10].

We will briefly present the extended SFM [10]: the social force model generates trajectories as if there were free particles in a 2D space, The model have the following formulation:

$$
\frac{d \boldsymbol{v}_{n}(t)}{d t} m=\boldsymbol{f}_{n}(t)
$$

subject to velocity and acceleration restrictions.

Assuming that a pedestrian tries to adapt his or her velocity $v_{n}$ within a relaxation time $k_{i}^{-1}$, the steering force

$$
\boldsymbol{f}_{n}^{\text {goal }}\left(\mathcal{D}_{n}\right)=k_{n}\left(\boldsymbol{v}_{n}^{0}\left(\mathcal{D}_{n}\right)-\boldsymbol{v}_{n}\right)
$$

where $\boldsymbol{v}_{n}^{0}\left(\mathcal{D}_{m}\right)$ is the desired velocity to reach its destination $\mathcal{D}_{n}$. The interaction forces are defined as follows:

$$
\boldsymbol{f}_{n, q}^{i n t}=a_{q} e^{\left(d_{q}-d_{n, q}\right) / b_{q}} \frac{\boldsymbol{d}_{n, q}}{d_{n, q}},
$$

where $q \in Q$, being $Q=P \cup O \cup R$ either a person, an object of the environment, or a robot.

Accordingly, the resultant force is calculated as a summation:

$$
\boldsymbol{f}_{n}(t)=\boldsymbol{f}_{n}^{\text {goal }}\left(\mathcal{D}_{n}\right)+\sum_{j \in P} \boldsymbol{f}_{n, j}^{i n t}+\sum_{o \in O} \boldsymbol{f}_{n, o}^{i n t}+\sum_{r \in R} \boldsymbol{f}_{n, r}^{i n t}
$$

where for each interaction force corresponds a set of force parameters $\theta=\left\{k, a_{n q}, b_{n q}, \lambda_{n q}, d_{n q}\right\}$. Instead, we propose an improvement of the model, by estimating a specific $\theta$ parameters for each pair of $q$ targets that better suit to the observed data and the situation.
In the remainder of the paper, as can be seen in Fig. 2, we will use the Intentionality Predictor (BHMIP), which serves to estimate the best destination a person aims to, and it is described in [10]. Although it is out of the scope discussing how the BHMIP works, it is used intensively throughout the present paper. The other modules of the scheme are the main contributions of the present paper: a behavior estimator and a trajectory prediction that makes use of the presented framework.

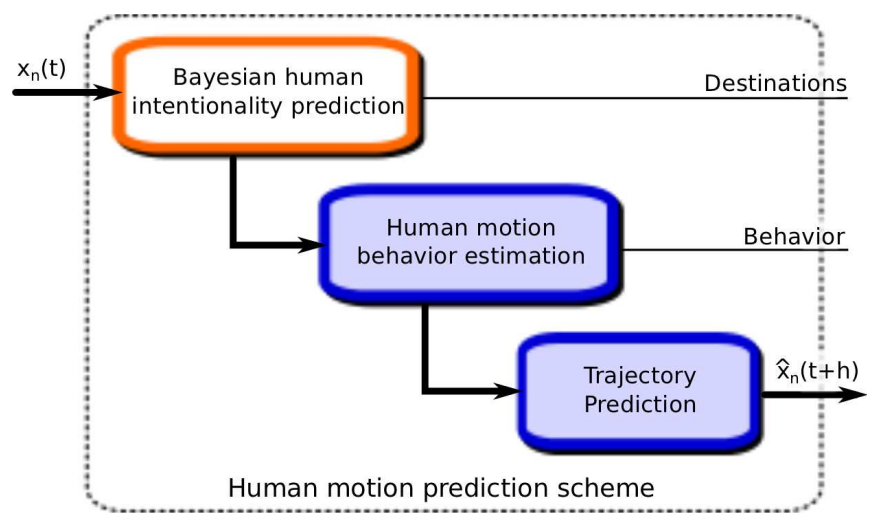

Fig. 2. Basic scheme of the proposed prediction algorithm and required estimators for a complete framework for human motion prediction.

\section{BEHAVIOR ESTIMATION}

During experimentation in real scenarios [11], [12] we observed a high variability in human motion and it served as the motivation to develop the present work. Given the high variability of human behavior, it is difficult to accurately predict human motion using the same set of parameters $\theta$, since there appear a clear difference between predictions and observations. Accordingly, our hypothesis is that each pair of interactions is characterized by an specific set of parameters $\theta$. Therefore, the prediction problem becomes an estimation of those specific behaviors $\theta_{l}$.

To this end, we define a set of possible behaviors $\boldsymbol{B}=$ $\left\{B_{1}, B_{2}, \ldots, B_{L}\right\}$, each of them directly corresponds to a set of interaction parameters $\theta_{l}=\left\{k_{l}, a_{l}, b_{l}, \lambda_{l}, d_{l}\right\}$, which define the interaction forces according to the SFM. Additionally, the inherent variable $\mathcal{B}_{n, q}$ is the estimated behavior between $n$ and $q$, and can be any $B_{l} \in \boldsymbol{B}$. Accordingly, we propose a method to infer the latent state $\mathcal{B}_{n}(t)=B_{l}$ of a person that describes its force interaction with its nearby targets, and hence, its trajectory.

The behavior $\mathcal{B}_{n, q}$ corresponds to the interaction between two persons. For instance, a person may react very conservative to an unexpected target while simultaneously behave passively with a companion. As depicted in Fig. 3, we can observe how different targets exert different forces which can only be explained if different behaviors are applied to each target.

The set of behaviors corresponding to one target is defined as $\mathcal{B}_{n}=\left\{\mathcal{B}_{n, q}, \quad \forall q \neq n\right\}$ as the set of parameters that describe the interactions of the $n$th target and its surrounding targets: 


$$
\boldsymbol{f}_{n}^{i n t}\left(\mathcal{B}_{n}\right)=\sum_{q \in Q} \boldsymbol{f}_{n, q}^{\text {int }}\left(\mathcal{B}_{n, q}\right)
$$

We propose to use a HMM algorithm to classify the sequential data information to infer the hidden behavior state:

$$
\begin{array}{r}
P\left(\mathcal{B}_{n, q}(t)=B_{l} \mid \boldsymbol{X}(t)\right)=\eta \cdot P\left(\boldsymbol{X}(t) \mid \mathcal{B}_{n, q}(t)\right) . \\
\sum_{\mathcal{B}_{n, q}(t-1)} P\left(\mathcal{B}_{n, q}(t) \mid \mathcal{B}_{n, q}(t-1)\right) P\left(\mathcal{B}_{n, q}(t-1) \mid \boldsymbol{X}(t-1)\right)
\end{array}
$$

where

$$
P\left(\boldsymbol{X}(t) \mid \mathcal{B}_{n j}(t)\right)=\mathcal{N}\left(\left\|\hat{\boldsymbol{f}}_{\text {obs }}^{\text {int }}-\boldsymbol{f}_{n}^{\text {int }}\left(\mathcal{B}_{n}\right)\right\| ; \mu_{f}, \Sigma_{f}\right)
$$

is a Gaussian distribution. The estimated interaction force is formulated as

$$
\hat{\boldsymbol{f}}_{\text {obs }}^{\text {int }}=\boldsymbol{f}_{\text {obs }}-\boldsymbol{f}_{n}^{\text {goal }}\left(\mathcal{D}_{n}\right) .
$$

We can calculate the observed force $f_{\text {obs }}$ following (3), and the force to goal $\boldsymbol{f}_{n}^{\text {goal }}\left(\mathcal{D}_{n}\right)$ by using (4), which is only possible after estimating the destination $\mathcal{D}_{n}$. The resulting subtraction is the estimated interaction force $\hat{\boldsymbol{f}}_{o b s}^{i n t}$.

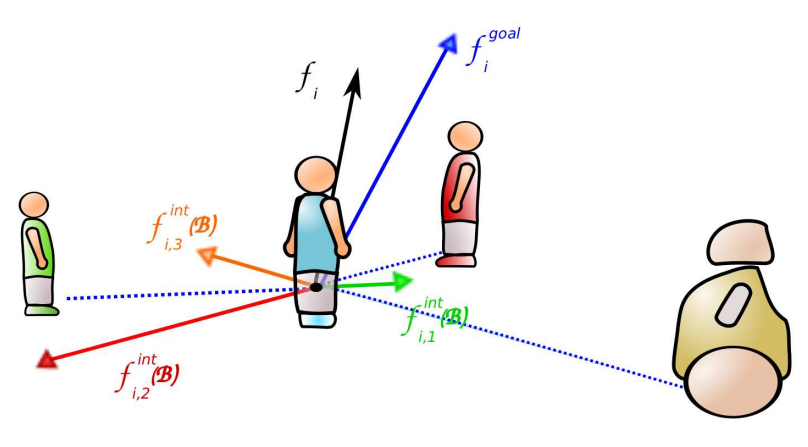

Fig. 3. Scheme of the social forces based on behavior estimations. As can be seen, the behavior $\mathcal{B}_{i}$ determines the magnitude of the forces, and hence the trajectories propagation.

\section{A. Behavior clustering}

We need to obtain certain knowledge about the values of the basic classes $\boldsymbol{B}$ in order to use the algorithm proposed. Consequently, the clustering problem boils down to obtain a large set of $\theta_{n q}=\left\{k, a_{n q}, b_{n q}, \lambda_{n q}, d_{n q}\right\}$ parameters, which are direct responsible for the motion behaviors as explained in this section.

We can obtain the estimated force of interaction $\hat{\boldsymbol{f}}_{\text {obs }}^{\text {int }}$ as described in (10). However, we now require a function or method to calculate the values of $\theta$ that fit better to the observation of the force $\hat{\boldsymbol{f}}_{\text {obs }}^{\text {int }}$. The training data provided assumes a one-at-a-time interaction of the target $n$ with a single target $q$ to facilitate the calculations.

$$
\hat{\theta}_{n, q}=\arg \min _{\theta}\left(\left\|\hat{\boldsymbol{f}}_{\text {obs }}^{\text {int }}-\boldsymbol{f}_{n, q}^{i n t}(\theta)\right\|^{2}\right)
$$

In general, any method to calculate this minimization solves the problem of finding the best $\hat{\theta}_{n, q}$. We have chosen a sampling method, the MCMC-MH, by making use of a priori information of values of typical $\theta$ parameters calculated in other works ([5], [4], [12]) to constrain the search space.

Once we have obtained a set of $\theta_{n, q}$ parameters (for simplification referred hereafter as $\theta_{k}$ ), we make use of the Expectation-Maximization algorithm to obtain the clusters of $\theta$, and thus, the behavior primitive classes $B_{l} \in \boldsymbol{B}$.

The Expectation is calculated as:

$$
\gamma\left(\theta_{k}\right)=\frac{\pi_{l} \mathcal{N}\left(\theta_{k} ; \mu_{l}, \Sigma_{l}\right)}{\sum_{j}^{L} \pi_{j} \mathcal{N}\left(\theta_{k} ; \mu_{j}, \Sigma_{j}\right)}
$$

and the Maximization:

$$
\begin{gathered}
\pi_{l}=\frac{\sum \gamma\left(\theta_{k}\right)}{\sum \gamma(\theta)} \\
\mu_{l}=\frac{\sum \gamma\left(\theta_{k}\right) \cdot \theta_{k}}{\sum \gamma\left(\theta_{k}\right)} \\
\Sigma_{l}=\frac{\sum \gamma\left(\theta_{k}\right) \cdot\left(\theta_{k}-\mu_{l}\right)\left(\theta_{k}-\mu_{l}\right)^{\top}}{\sum \gamma\left(\theta_{k}\right)}
\end{gathered}
$$

As we will demonstrate in Section V, the dimensionality of the SFM parameters $\theta$ can be reduced since the most significant variables are $\{a, b\}$ for our experiment, which greatly reduces the complexity of this calculation.

\section{MULTI-HYPOTHESIS MOTION PREDICTION}

This section, as explained at the beginning of the paper, makes use of the above explained tools and methods to characterize typically human behaviors, and proposes a straightforward algorithm to integrate all the presented parts into a framework for enhancing the human motion prediction.

Intentionality prediction is essential for a good understanding of human motion. We require destinations as explained in previous sections, to set points in a scene as goal-interesting places. Behavior estimation is also of vital importance for the correct prediction of human trajectories. Therefore, we will update the probabilities $P\left(\mathcal{B}_{n, q}(t)=B_{l} \mid \boldsymbol{X}(t)\right)$ and $P\left(\mathcal{D}_{n}(t)=D_{m} \mid \boldsymbol{X}_{n}(t)\right)$ at every instant $t$. For simplicity of the explanation, we assume that the most expectable $\mathcal{D}_{n}(t)$ and $\mathcal{B}_{n, q}(t)$ are the only destinations' estimations kept. We will explain later a multi-hypothesis prediction method that contemplates different outcomes for a given scene configuration.

Accordingly, the estimations of $\mathcal{D}_{n}(t)$ and $\mathcal{B}_{n, q}(t)$ are available throughout the remaining calculations, facilitating the prediction of human motion.

Let $\boldsymbol{s}_{n}(t)=\left[\boldsymbol{x}_{n}(t), \boldsymbol{v}_{n}(t)\right]^{\top}$ be the augmented state vector for the $n$th person in the scene consisting of position and its derivate $\boldsymbol{v}_{n}(t)=\dot{\mathbf{x}}_{\mathbf{n}}(t)$. The motion propagation model is subject to the following differential equations:

$$
\dot{\boldsymbol{s}}_{n}(t)=d c\left(\boldsymbol{s}_{n}(t), \boldsymbol{f}_{n}\left(t, \mathcal{D}_{n}, \mathcal{B}_{n}\right)\right),
$$




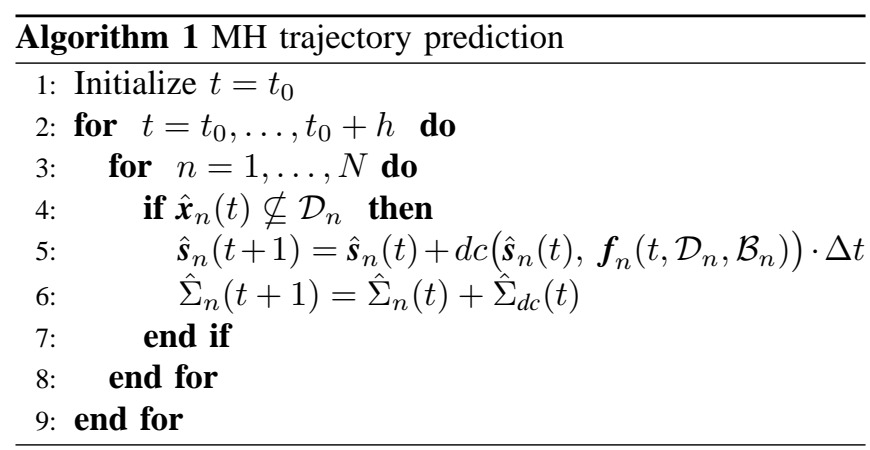

which requires as inputs its own state $\boldsymbol{s}_{n}(t)$ and the social forces $\boldsymbol{f}_{n}\left(t, \mathcal{D}_{n}, \mathcal{B}_{n}\right)$ which can be calculated as explained in (6). In the present paper, $d c\left(\boldsymbol{s}_{n}(t), \boldsymbol{f}_{n}\left(t, \mathcal{D}_{n}, \mathcal{B}_{n}\right)\right)=$ $\left[\boldsymbol{v}_{n}(t), \boldsymbol{f}_{n}\left(t, \mathcal{D}_{n}, \mathcal{B}_{n}\right)\right]^{\top}$.

Finally, we formulate a prediction trajectory for a time horizon of $h$ and its correspondent covariance. Let

$$
\hat{\boldsymbol{X}}_{n}(t+h)=\left\{\hat{\boldsymbol{x}}_{n}(t+1), \hat{\boldsymbol{x}}_{n}(t+2), \ldots, \hat{\boldsymbol{x}}_{n}(t+h)\right\}
$$

be the set of predicted positions and its corresponding augmented vector $\hat{\boldsymbol{s}}_{n}=\left[\hat{\boldsymbol{x}}_{n}, \hat{\boldsymbol{v}}_{n}\right]$ of positions and velocities:

$$
\begin{gathered}
\hat{\boldsymbol{S}}_{n}(t+h)=\left\{\hat{\boldsymbol{s}}_{n}(t+1), \hat{\boldsymbol{s}}_{n}(t+2), \ldots, \hat{\boldsymbol{s}}_{n}(t+h)\right\} \\
\hat{\Sigma}_{n}(t+h)=\left\{\hat{\Sigma}_{n}(t+1), \hat{\Sigma}_{n}(t+2), \ldots, \hat{\Sigma}_{n}(t+h)\right\} .
\end{gathered}
$$

As shown in Algorithm 1, all the present targets in the scene propagate simultaneously and the expected propagations are utilized in the next iteration. Once a target succeeds on its intention to reach its destination $\mathcal{D}_{n}$, it remains idle waiting for the rest of the targets to get to their corresponding destinations or until the time horizon $h$ expires.

An Euler integration method is chosen for the propagation of the ODE subject to a limitation in velocity $\|v\|<$ $v_{\max }$. The covariance $\hat{\Sigma}_{d c}(t)$ is calculated depending on the propagation values.

Regarding the multi-hypothesis issue, we should consider that the estimation of the target intentionality (see [10]) is not perfectly estimated and uncertainty is associated to other possible candidates for the best expected destination. As can be seen in Fig. 4, the target can move towards any of the $m$ th destinations in the scene, with probability $P\left(\mathcal{D}_{n}(t)=\right.$ $\left.D_{m} \mid \boldsymbol{X}_{n}(t)\right)$. A hypothesis $l$ is the set of combinations of people intentions, where each person has associated a single expected intentionality $\mathcal{D}_{n}=D_{l_{n}}$. Thus, the joint probability for $N$ persons is

$$
P\left(\mathcal{D}_{1}=D_{l_{1}}, \ldots, \mathcal{D}_{N}=D_{l_{N}} \mid \boldsymbol{X}\right)=\prod_{n \in N} P\left(\mathcal{D}_{n}=D_{l_{n}} \mid \boldsymbol{X}\right),
$$

where for each hypothesis $l$ the prediction is performed following algorithm 1. If there are many targets in the scene, the number of hypothesis grows exponentially and we should branch and prune the hypothesis to those whose joint probabilities of (20) are more expectable, and proceed to apply algorithm 1 with their respective parameters associated with each hypothesis.

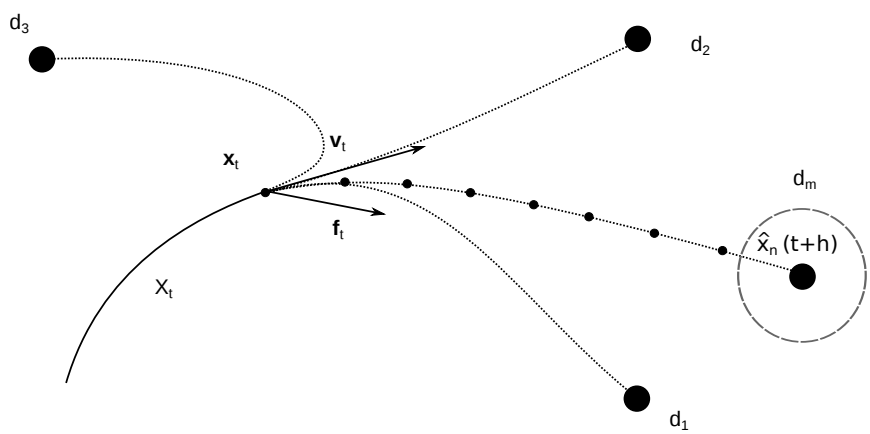

Fig. 4. Trajectory propagation as a result of the multi-hypothesis human motion prediction.

\section{EXPERIMENTS}

In order to conduct all the experiments and to test the presented approach, we have used a mobile service robot, developed for the URUS project [13], designed to work in urban pedestrian areas and to interact with people (see Fig. 1).

The experimental areas are the BRL (Barcelona Robot Lab) and the FME (Facultat de Matemàtiques i Estadística). Both environments are outdoor and urban, and are located respectively at the North and South Campus of the Universitat Politècnica de Catalunya (UPC). The BRL is a large section of the campus that was outfitted as an experimental area, covering over $10.000 \mathrm{~m}^{2}$, including six buildings and a square.

For the recording of the experiments, we have used two Hokuyo UTM-30LX 2D laser range sensors. Human detection is done using laser information and the tracker helps out to maintain targets in the scene (for further details, see [12]).

The first part of the experiments, performed in the FME, consisted of one robot and one person as obstacles and a set of volunteers performed experiments in a controlled scene. The objective was obtaining data to calculate the behavior classes, as explained in Section III-A. Over 40 volunteers were recorded during a full day of experiments. Men and women, ranging from 20 to 56 years old participated as volunteers and some of them had not any experience in robotics.

The experiment setting was simple: the volunteers were told to naturally walk towards a very visible destination (a huge red pylon), and meanwhile they approached their destination, first the person-obstacle and then the robot, moved crossing his/her path. During the experiment, it was very important not to interact with both obstacles at the same time, but the volunteers were not told anything regarding this condition.

Once obtained and processed the trajectories, the parameters $\theta_{k}=\left\{k, a_{k}, b_{k}, \lambda_{k}, d_{k}\right\}$ can be obtained by simply applying the procedure described in Sec. III-A. As commented before, we have reduced the dimensionality of the SFM parameters $\theta$ to the $\{a, b\}$ parameters only for plotting purposes. A PCA analysis revealed that the two principal eigenvectors, were almost a linear combination of $\{a, b\}$ exclusively and their respective eigenvalues concentrated more than $99 \%$ of the "energy". 


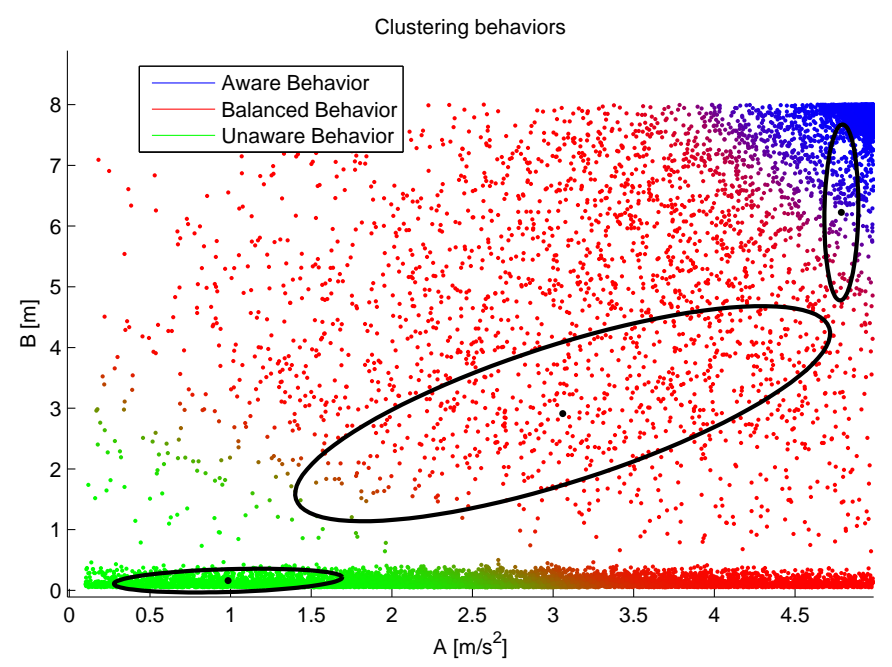

Fig. 5. Cluster of behaviors, represented on the parameters $\{a, b\}$.

The results in Fig. 5 clearly show three basic behaviors. The Aware behavior, in blue, $\{k=2.3, a=4.78, b=$ $6.22, \lambda=0.56, d=0.20\}$ is high both in $a$ and $b$ and represents all those trajectories of people extremely cautious, that exceed in consideration towards the other target. This behavior was clearly visible when some volunteers seemed to be afraid of the robot.

In green, the Unaware behavior $\{k=2.3, a=0.98, b=$ $0.16, \lambda=0.56, d=0.20\}$, corresponding to all those people that virtually ignored their targets obstacle and went to their destination no matter what. This behavior is not atypical and it may happen often in daily life.

And finally, in red, we can observe the Balanced behavior $\{k=2.3, a=3.05, b=2.91, \lambda=0.56, d=0.20\}$. This cluster describes all those behaviors that are not Aware or Unaware behaviors, and hence, it is a mixture of a great variety of behaviors.

The separability of clusters is not really significant, but this classification answers most of the questions arisen regarding the interaction of moving targets in a scene. It is not important the nature of the target, but the consideration of the person towards the target. An implication of this paradigm of interaction modeling is that it is no longer reciprocal, that is, an Unaware pedestrian may inflict great social-force stress to other pedestrians, but in response he or she gets almost no social-force feedback since the force exerted by other people to the Unaware pedestrian is much more shorter. On the contrary, an Aware pedestrian may suffer more social stress on a typically social environment. All those considerations greatly affect the deployment of social robots, being the first, the Unaware target a threaten to the integrity of the robot, and the Aware pedestrian would suffer a high social stress in the presence of a robot which should act accordingly to reduce that impact. For these reasons it is of vital importance to detect those behaviors if we want to deploy social robots on urban or social environments and being accepted by humans and facilitate their daily life and not in the contrary.

In Fig. 6 is depicted a function of the social-force module $\left|\hat{\boldsymbol{f}}_{\text {obs }}^{\text {int }}\right|$ for each set of parameters with respect to distance to

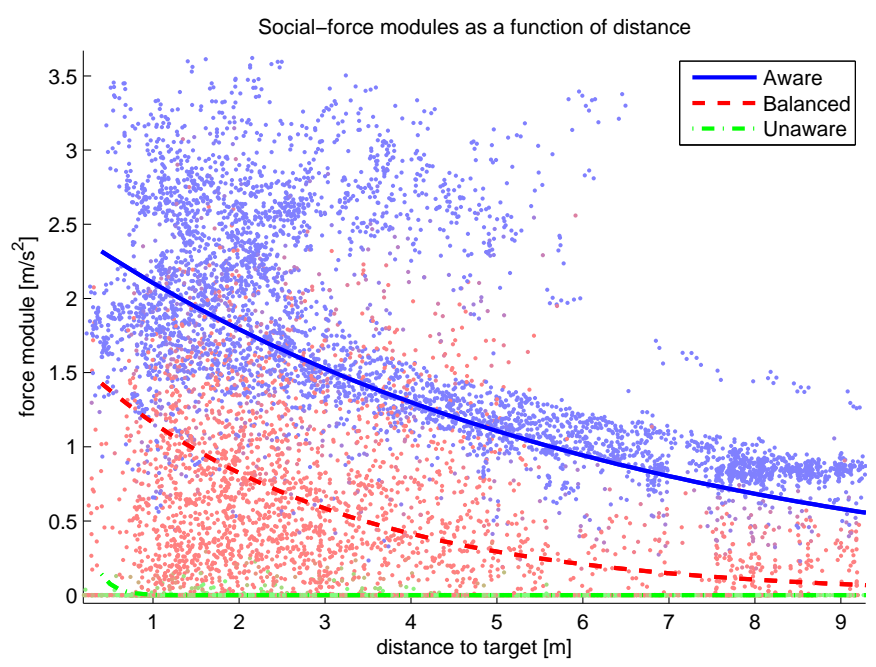

Fig. 6. Social-force module $\left|\hat{\boldsymbol{f}}_{o b s}^{i n t}\right|$ with respect to distance from interacting target.

target. Additionally, we can observe the obtained social-force parameters for each $\theta_{k}$ that resulted in the minimization of (11). We can appreciate that the separation of the clusters is not so significant, despite the noisy conditions and the variability of humans.

The second part of the experiments was performed in the BRL. Using the parameters obtained before for each behavior, we tested the algorithm during a full morning of experiments. This time, the experiment setting was even more simple. We did not require any direct volunteer: the robot was navigating in the campus and meanwhile recording trajectories of persons on their natural habitat.

The idea was to apply the algorithms explained before in a real scenario where nobody knows which kind of experiment the robot is performing. For all the trajectories observed, we have calculated the most expectable destination every target aims to.

An example of an interaction is depicted in Fig. 7. The robot slowly moves and three students quickly walk to attend a class. Each of the targets is depicted in the same color on each picture. On the left picture, the algorithm predicts that target green may stop, due to the interaction with the blue target and the robot. As can be seen in the complete trajectory, this never happens and green simply changes its direction and this time the algorithm predicts correctly. All those prediction trajectories, and their corresponding covariances are calculated on a time horizon of two seconds.

In order to obtain reliable results and not a single experiment of prediction, we have tested under the following circumstances. For an incremental time horizon up to 10 seconds, we have evaluated the performance of the prediction algorithm for each time of the interval, that is, starting just after the observation on a horizon of 0.1 seconds to the maximum horizon of 10 seconds.

In order to evaluate the accuracy of a prediction, we have set a binary function which returns a successful prediction if the prediction at time $t+h$ is within 1 meter of the real trajectory performed by the target. Additionally, we 


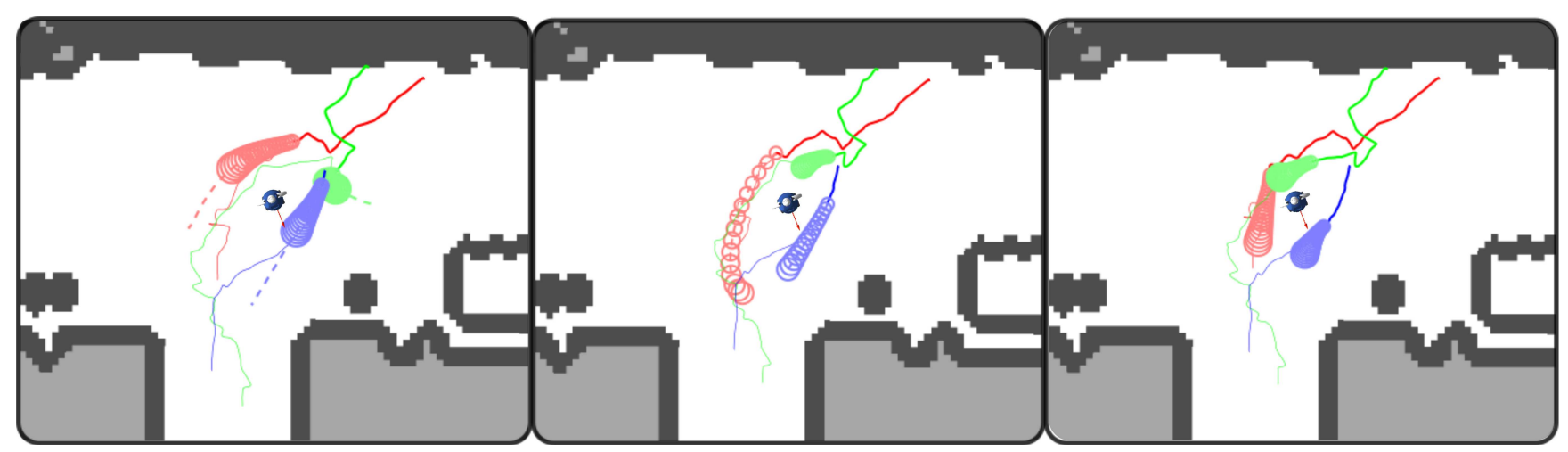

Fig. 7. Prediction example. Each of the targets is depicted in the same color on each picture. On the left picture, the algorithm predicts that target green may stop, due to the interaction of the blue target and the robot. All those prediction trajectories, and their corresponding covariances are calculated on a time horizon of two seconds.

have compared various methods. As can be seen in Fig. 8, in dashed blue is depicted the linear propagation as a prediction method. This method simply filters the velocity and propagates accordingly to the remaining horizon time. In solid green is depicted the performance of the social-force approach, that is, making use of intentionality information and interaction forces, but using fixed parameters. It clearly outperforms the linear propagation, even for a short horizon time and for long horizons, it simply can not be compared. In dashed dotted red is drawn our complete approach, now using prediction information as well as behavior estimation. We can appreciate how the performance can be enhanced just by adding intentionality prediction and behavior information with respect to the linear propagation.

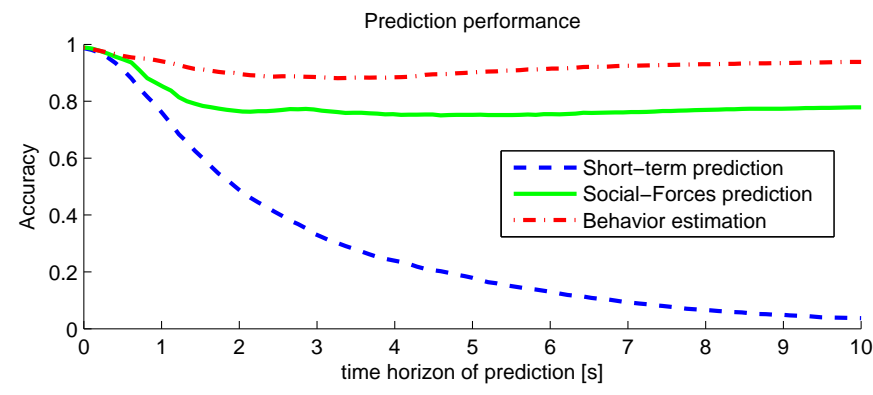

Fig. 8. Performance function of the proposed method, a comparison with a short-term predictor, and a prediction only based on forces.

On average, the performance of the short-term velocity propagation method is $28.66 \%$, for the social-force $78.47 \%$, which only uses intentionality information, and a $91.71 \%$ accuracy for the proposed method using behavior estimation and intentionality information.

\section{CONCLUSION AND FUTURE WORK}

In this paper we have presented a complete framework of human motion prediction in social environments. The framework consists of a prediction algorithm, the intentionality predictor (BHMIP), and the behavior estimator proposed in this work. We have demonstrated the usefulness of the proposed framework, and how the integration of intentionality prediction and motion behavior can greatly enhance the performance of the prediction algorithm.
In addition, we have presented a robust estimator of behavior, where three different basic behaviors have been detected: Aware, Balanced and Unaware. Real experimentation has demonstrated that the integration of the proposed prediction tools clearly improves the accuracy of human motion prediction in real scenarios.

\section{REFERENCES}

[1] D. Helbing and P. Molnar, "Social force model for pedestrian dynamics," Physical review E, vol. 51, no. 5, pp. 4282-4286, 1995.

[2] M. Kuderer, H. Kretzschmar, C. Sprunk, and W. Burgard, "Featurebased prediction of trajectories for socially compliant navigation," in Proc. of Robotics: Science and Systems (RSS), 2012.

[3] M. Moussaï,, D. Helbing, and G. Theraulaz, "How simple rules determine pedestrian behavior and crowd disasters," Proceedings of the National Academy of Sciences, vol. 108, no. 17, pp. 6884-6888, 2011.

[4] M. Luber, L. Spinello, J. Silva, and K. O. Arras, "Socially-aware robot navigation: A learning approach," in Proceedings of the IEEE/RSJ International Conference on Intelligent Robots and Systems, 2012, pp. 902-907.

[5] F. Zanlungo, T. Ikeda, and T. Kanda, "Social force model with explicit collision prediction," EPL (Europhysics Letters), vol. 93, no. 6, 2011.

[6] G. Arechavaleta, J.-P. Laumond, H. Hicheur, and A. Berthoz, "An optimality principle governing human walking," IEEE Transactions on Robotics, vol. 24, no. 1, pp. 5-14, 2008.

[7] M. Bennewitz, W. Burgard, G. Cielniak, and S. Thrun, "Learning motion patterns of people for compliant robot motion," The International Journal of Robotics Research, vol. 24, no. 1, pp. 31-48, 2005.

[8] A. Foka and P. Trahanias, "Probabilistic Autonomous Robot Navigation in Dynamic Environments with Human Motion Prediction," International Journal of Social Robotics, vol. 2, no. 1, pp. 79-94, 2010.

[9] B. Ziebart, N. Ratliff, G. Gallagher, C. Mertz, K. Peterson, J. Bagnell, M. Hebert, A. Dey, and S. Srinivasa, "Planning-based prediction for pedestrians," in Proceedings of the IEEE/RSJ International Conference on Intelligent Robots and Systems, 2009, pp. 3931-3936.

[10] G. Ferrer and A. Sanfeliu, "Bayesian human motion intentionality prediction in urban environments," Pattern Recognition Letters, no. 0, pp. -, 2013. [Online]. Available: http://www.sciencedirect.com/science/article/pii/S0167865513003176

[11] G. Ferrer, A. Garrell, and A. Sanfeliu, "Social-awareness robot navigation in urban environments," in European Conference on Mobile Robotics, ECMR., 2013.

[12] _ , "Robot companion: A social-force based approach with human awareness-navigation in crowded environments," in Proceedings of the IEEE/RSJ International Conference on Intelligent Robots and Systems, 2013, pp. 1688-1694.

[13] E. Trulls, A. Corominas Murtra, J. Pérez-Ibarz, G. Ferrer, D. Vasquez, J. Mirats-Tur, and A. Sanfeliu, "Autonomous navigation for mobile service robots in urban pedestrian environments," Journal of Field Robotics, vol. 28, no. 3, pp. 329-354, 2011. 